\title{
Direct imprinting using soft mold and gas pressure for large area and curved surfaces
}

\author{
Jer-Haur Chang, Fang-Sung Cheng, Chi-Chung Chao, \\ Yung-Chun Weng, and Sen-Yeu Yang ${ }^{\text {a) }}$ \\ Grace Laboratory for Polymer Processing, Department of Mechanical Engineering, \\ National Taiwan University, Taipei, Taiwan 106, Republic of China \\ Lon A. Wang \\ Department of Electrical Engineering and Institute of Electro-Optical Engineering, National Taiwan \\ University, Taipei, Taiwan 106, Republic of China
}

(Received 29 April 2005; accepted 15 August 2005; published 25 October 2005)

\begin{abstract}
In this paper we report a simple and effective method that renders direct imprinting of sub-micron structures onto PMMA resist coated on large area and curved substrates using the PDMS mold on a closed chamber. Nitrogen gas was employed to generate a uniform pressure. The patterns of the soft mold could be replicated with high quality over an entire $12 \mathrm{in.} \mathrm{resist-coated} \mathrm{area.} \mathrm{The} \mathrm{process}$ was further successfully applied to the imprinting of a curved substrate. (C) 2005 American Vacuum Society. [DOI: 10.1116/1.2073447]
\end{abstract}

\section{INTRODUCTION}

Several imprint lithography techniques have been developed for the low cost and high resolution. They are nanoimprint lithography (NIL), ${ }^{1,2}$ soft lithography, ${ }^{3,4}$ ultraviolet (UV)-based NIL, ${ }^{5,6}$ and step and flash imprint lithography $(\mathrm{SFIL})^{7}$ et al. Recently, large area imprinting has gained considerable attention due to its enhanced productivity. Bailey et $a l .{ }^{8}$ has demonstrated $8 \mathrm{in}$. imprinting based on a SFIL process. Gourgon et al. ${ }^{9}$ have presented an 8 in. silicon wafer imprinted by the NIL process. No study has carried out an investigation for the imprinting of $12 \mathrm{in.} \mathrm{microstructures,}$ even though microstructures on a 12 in. silicon wafer is getting popular, and demand for such large-area one-step imprinting will be great soon. Our objective in this study is to construct a system that can achieve one-step large-area imprinting. Two problems have to be overcome: uniform contacting and uniform pressuring. Conventional mold materials such as silicons or quartzes may cause a poor contact problem, especially when the area is large or the resist is thin. Soft lithography has proposed elastomer mold techniques to achieve the conformal contact with the surface of interest. Soft mold will be used in this study. For overcoming the problem of uniform pressuring, which is important to largearea imprinting, Chang ${ }^{10}$ used a gas pressure mechanism for hot embossing. Microstructures on a 4 in. silicon wafer have been successfully replicated onto PC films. An air cushion press (ACP) was utilized at Nanonex Corportation system for NIL. ${ }^{11}$ With soft mold and gas pressure, one-step direct imprinting of microstructures onto resist on a 12 in. silicon wafer will be achieved.

The replication of submicron patterns onto a curved surface is another recent interest. Curved surfaces are difficult to pattern using conventional rigid-mold NIL and UV NIL. Ruchhhoeft et al. ${ }^{12}$ combined SFIL with ion beam proximity

a) Author to whom correspondence should be addressed; electronic mail: syyang@ntu.edu.tw printing to generate features on a silicon sphere ( 1 in. diameter). Accurate and expensive equipment was used. Whitesides et al. ${ }^{13}$ used a PDMS mold as an elastomeric phase mask to pattern nanostructures on a hemisphere surface (3/4-inch diameter) with features as small as $175 \mathrm{~nm}$. Choi ${ }^{14}$ formed submicron features on a polymer-coated spherical (20 mm diameter) substrate via hot embossing with a PDMS film stamp. So far, the area is small, and pressures were exerted by hand to keep substrates and soft mold in contact. To enhance close contacting and uniform pressuring during curved surface imprinting, soft mold and gas pressure are employed in this study.

\section{EXPERIMENTAL SETUP}

\section{A. Imprint apparatus and process}

A conventional hot press was used to carry out the imprinting, as illustrated in Fig. 1. However, the press was not used for pressuring the mold/substrate stack. It was used to clamp and seal a closed chamber. Nitrogen was used as the working gas to pressuring the stack, after the mold and substrate were heated by the block of the press. The pressure system is composed of a nitrogen tank $\left(120 \mathrm{Kg} / \mathrm{cm}^{2} \max .\right)$, valves, and a pressure regulator.

The proposed gas-assisted imprinting process includes four steps: (a) placing the substrate with PMMA resist onto the mold to form a substrate-mold stack, which is then placed above the heating plate; (b) covering the substratemold stack with a seal (polyester, PET) film and enclosing the stack in the chamber; (c) introducing the nitrogen gas into the chamber to heat and press the stack; (d) cooling the stack, exhausting the nitrogen, and finally opening the chamber to retrieve the imprinted substrate.

\section{B. Preparation of large area PDMS molds}

In this study, patterns on the 12 in. PDMS mold were obtained by assembling patterns from a 4 in. silicon mold, on 


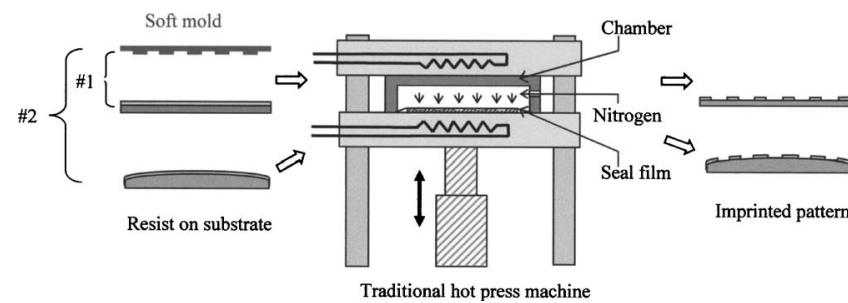

FIG. 1. Schematic of the gas pressurized apparatus and imprinting process for a 12 in. silicon wafer and a 2 in. curved substrate imprinted. \#1, process for planar imprinting; \#2, process for nonplanar imprinting

which are several types of patterns with feature sizes ranging from $600 \mathrm{~nm}$ to $20 \mu \mathrm{m}$. The patterns on the master mold was replicated onto polycarbonate (PC) films using a gasassisted hot embossing process. The patterned PC films were then trimmed, assembled, and glued to a glass surface to form a 12 in. PC film, which served as a transmitter mold. The 12 in. PDMS molds were then prepared by casting polydimethylsiloxane (PDMS) (Sylgard ${ }^{\mathrm{TM}}$ 184, Dow Corning) at a mix ratio of 10:1 on the transmitter mold. The formed mold was cured at room temperature for one day. The replica patterns of the PDMS mold thus contains multiple microstructures. The depth is $350 \mathrm{~nm}$. The fabrication procedure of the 12 in. PDMS mold is illustrated in Figs. 2(a)-2(d). The scan- (a) Pattern on 4 in. silicon mold

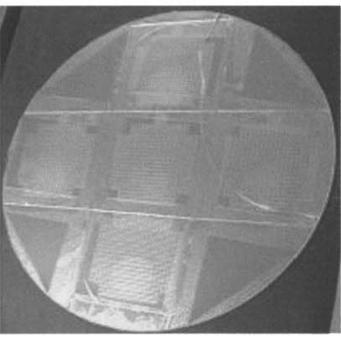

(c) Assembled patterns on 12 in.PC film

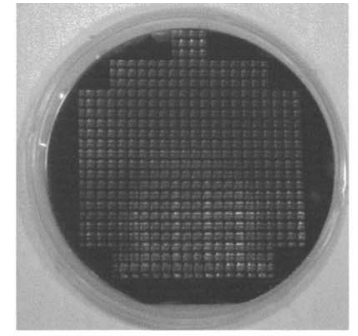

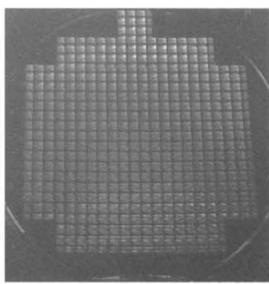

(b) Replica of patterns on 4 in. PC film

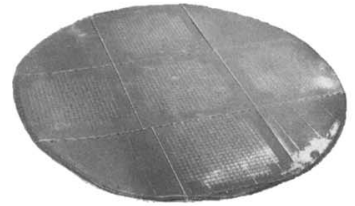

(d) Replica of pattern on 12 in.PDMS mold

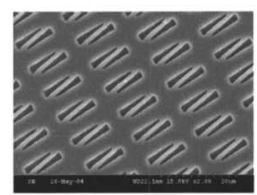

Patterns on silicon mold

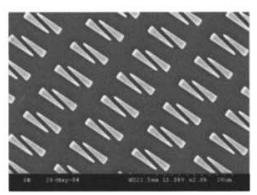

Patterns on PC film

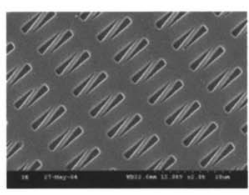

Patterns on PDMS mold (e)

FIG. 2. Photograph of a 12 in. PDMS mold fabricated procedure: (a) 4 in. silicon mold with a series of patterned squares as a master mold; (b) PC film hot embossing; (c) assembled PC films up to a 12 in. diameter; (d) 12 in. PDMS mold casting; (e) SEM images on surface of silicon mold, PC film, and PDMS mold. ning electron microscope (SEM) images on the surfaces of silicon mold, PC film, and PDMS mold are shown in Fig. 2(e). The features shown were triangles with the base of $2 \mu \mathrm{m}$ and a height of $8 \mu \mathrm{m}$. The sharp-pointed sizes were smaller than $600 \mathrm{~nm}$. The PDMS mold was of the same shape patterns and quality as the silicon mold. For fabricating PDMS of a very large area, the proposed system is simple, the cost is low, and the production is fast.

In this paper, a support ring used to support PDMS mold can overcome distortion. The rigid outer support ring should match the outward lateral geometry of the PDMS mold with a height equal to or less than that of the PDMS mold.

\section{Preparation of imprint substrates and resist}

In the experiment, two different types of substrates imprinted were used: the resists on a 12 in. silicon wafer and on 2 in. curved stainless steel with a 3 in. radius of curvature. The curved stainless steel was machined and polished. The polymeric resist was prepared from $12 \mathrm{wt} \%$ of polymethylmethacrylate (PMMA, molecular weight 120K, Aldrich) solution in toluene. The glass transition temperature $\left(T_{g}\right)$ was around $114{ }^{\circ} \mathrm{C}$. Both substrates were dip coated in the PMMA resist and then baked on a contact hotplate for $5 \mathrm{~min}$ at $150{ }^{\circ} \mathrm{C}$ to remove the residual solvent.

\section{Imprint conditions}

The imprinting temperature, the time of imprinting, and the applied gas pressure are chosen such that the polymer completely fills the cavities of the PDMS mold during imprinting. After investigation experiments were carried out at an imprinting temperature of $180{ }^{\circ} \mathrm{C}$ with a gas pressure of $40 \mathrm{Kg} / \mathrm{cm}^{2}$, and with the pressing time of $5 \mathrm{~min}$.

\section{RESULTS}

\section{A. Pressure uniformity}

Pressure uniformity over a large area was investigated with pressure-sensitive films. The pressure sensitive film (Fuji Prescale Film, Japan) can indicate pressures by colors from 25 to $100 \mathrm{Kg} / \mathrm{cm}^{2}$. The pressure-sensitive film (15 $\times 15 \mathrm{~cm}^{2}$ ) was sandwiched between an 8 in. PDMS mold and silicon wafer under the gas pressure of $45 \mathrm{Kg} / \mathrm{cm}^{2}$. Color density of the pressed film was measured by a densitometer detector and the result was shown in Fig. 3(a). The measured pressure values over the imprint area were $43 \pm 3 \mathrm{Kg} / \mathrm{cm}^{2}$, as shown in Fig. 3(b). This proves that the PDMS mold and gas pressure mechanism can achieve the close contact and uniform pressing demonstrated for onestep direct imprinting on a large area substrate.

\section{B. 12 in. silicon wafer imprinted}

The first time 12 in. silicon wafer fully one-step imprinting has been achieved using the proposed process is shown in Fig. 4(a). It also can be seen imprinting uniformity around the wafer for a given pattern, as shown in Fig. 4(b). The patterns were constructed with a pitch of $3.2 \mu \mathrm{m}$ and a width 


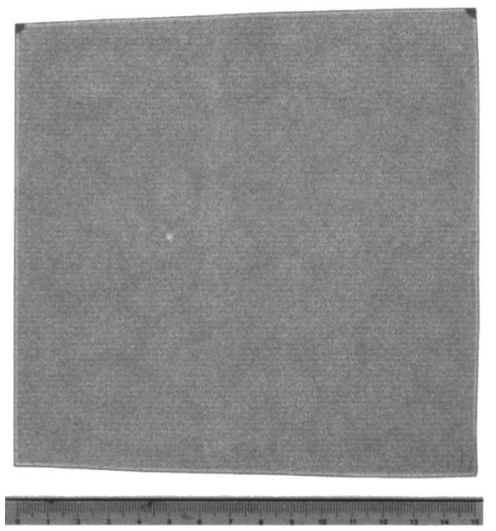

(a)

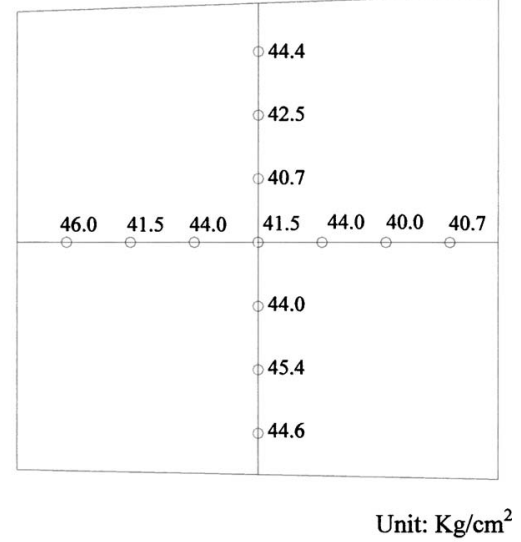

(b)
FIG. 3. (a) Photograph of indicating papers (15 $\times 15 \mathrm{~cm}^{2}$ ) under the gas pressure of $45 \mathrm{~kg} / \mathrm{cm}^{2}$; (b) the measured pressure values over the imprint area were $43 \pm 3 \mathrm{~kg} / \mathrm{cm}^{2}$. of $1.6 \mu \mathrm{m}$. The SEM images show that pattern structures were almost identical and defect-free for all the fields. In addition, the atomic force microscope (AFM) images are shown in Fig. 4(c). The line structures on the PDMS mold and resist surfaces with opposite patterns at the same position are illustrated, displaying the width of $1.6 \mu \mathrm{m}$, depth of $350 \mathrm{~nm}$, and pitch of $3.2 \mu \mathrm{m}$. By using a mold made from PDMS, owing to the low interfacial free energy $(22-24 \mathrm{dyn} / \mathrm{cm})$, it can be easily released without damaging the resist; ${ }^{3}$ the pattern distortion can be significantly reduced due to the similar coefficient of thermal expansion between mold and resist. This indicates a success of imprinting process. The patterns were successfully transferred by one imprinting step on the entire wafer surface. These results show that the presented technology with the PDMS and the pressure system is good enough to duplicate the patterns on a very large surface. Note that the linewidth of $1.6 \mu \mathrm{m}$ on the master mold is not the smallest scale for the soft mold and gas pressure mechanism imprinting. In fact, the resolution can be further scaled below $1.6 \mu \mathrm{m}$ by our fabricated process.

\section{Nonplanar substrate imprinted}

Figure 5(a) shows a photograph of a 2 in. curved substrate with a 3 in. radius of curvature imprinted using a gas pressure mechanism and a PDMS mold. Figure 5(b) shows the imprinted patterns on the curved surface. An optical microscope (OM) generated by a focusing microscope on the part of the sloping surface that lies in the center of the field of view. This image highlights the limited depth of field associated with conventional photolithographic methods. Due to the PDMS mold's elastomeric character, the PDMS mold conforms its shape to the substrate surface, thus generating the smooth contact. These patterns were successfully reproduced with a good shape pattern.

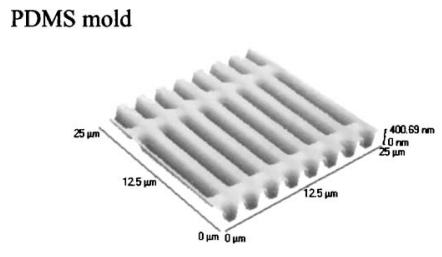

PMMA resist

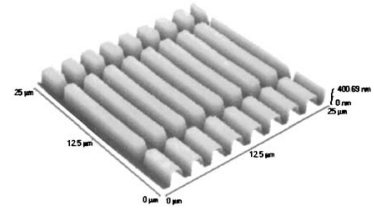

(c)

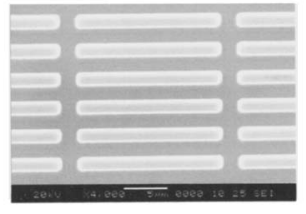

top

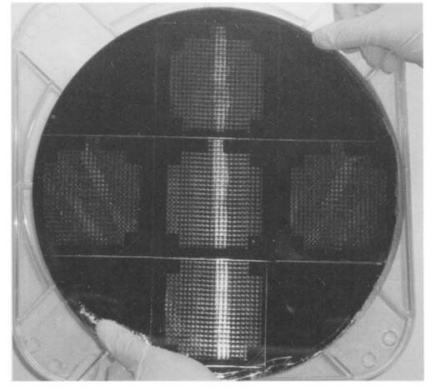

(a) 12 in. imprinted wafer
FIG. 4. (a) Photograph of a 12 in. imprinted wafer; (b) SEM images of three line patterns from a 12 in. silicon wafer, the minima feature size of $1.6 \mu \mathrm{m}$ width; (c) AFM images of a line with microstructures of $1.6 \mu \mathrm{m}$ width and $3.2 \mu \mathrm{m}$ pitch with $350 \mathrm{~nm}$ depth. 


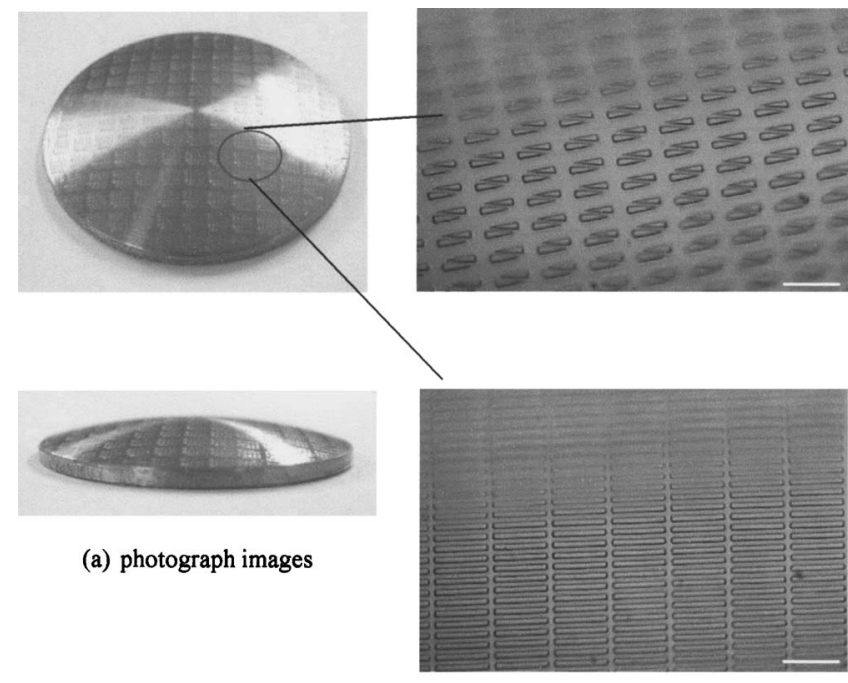

(b) OM images, scale bar is $15 \mu \mathrm{m}$

FIG. 5. Nonplanar surface imprinted: (a) photograph of a 2 in. curved stainless steel with a 3 in. radius of curvature; (b) OM images of patterns of line and triangular on a curved surface.

\section{CONCLUSIONS}

From these results, the comparison of the traditional platen pressing and gas pressing with a soft mold process can be shown as Table I. It is confirmed that we can fabricate correctly patterns on a 12 in. silicon wafer and a 2 in. curved substrate in one imprint step without causing any defect using a PDMS mold and gas pressure mechanism. Because the uniformity of the pressure was applied to perform imprinting in our fabricated process, the feature sizes of patterns were also consistent under the imprinting conditions.

TABLE I. A comparison of the traditional platen pressing and gas pressing with soft mold process.

\begin{tabular}{lcc}
\hline \hline & Platen pressing & $\begin{array}{c}\text { Gas pressing } \\
\text { with soft mold }\end{array}$ \\
\hline Pressure distribution & Nonuniform & Excellent uniform \\
Imprinting area & Small & Very large \\
Tool material & Hard & Hard/Soft \\
Substrate shape & Planar & Planar/Curve \\
UV exposure & Difficulty & Easy \\
Cost & High & Low \\
\hline \hline
\end{tabular}

\section{SUMMARY}

In this paper we present an innovative method and a mechanism for a very large area imprinting using gas pressure to press the soft mold and substrate. Replications of submicron features on a 12 in. silicon wafer and a 2 in. curved substrate in one step were demonstrated. SEM and AFM observations show uniform and clear transferred patterns into the PMMA resist. The high fidelity of feature replication implies that the new process has its potential as a low-cost one-step direct large area imprinting for enhanced productivity.

\section{ACKNOWLEDGMENTS}

This work was supported by the National Science Council under Contract No. NSC 93-2218-E-002-017. Partial financial support was also granted by the MLI Company. Special thanks to the Formosa Komatsu Silicon Corporation for the 12 in. silicon wafer supply were appreciated. We also thank Professor Lo for carrying out the AFM measure and coworkers in the Grace Laboratory for providing experimental assistance for polymer processing.

${ }^{1}$ S. Y. Chou, P. R. Krauss, and P. J. Renstrom, Appl. Phys. Lett. 67, 3114 (1995).

${ }^{2}$ S. Y. Chou, P. R. Krauss, W. Zhang, L. Guo, and L. Zhuang, J. Vac. Sci. Technol. A 15, 2897 (1997).

${ }^{3}$ J. L. Wilbur, A. Kumar, E. Kim, and G. M. Whitesides, Adv. Mater. (Weinheim, Ger.) 6, 601 (1994).

${ }^{4}$ Y. Xia and G. M. Whitesides, Annu. Rev. Mater. Sci. 28, 153 (1998).

${ }^{5}$ B. Vratzov, A. Fuchs, M. Lemme, W. Henschel, and H. Kurz, J. Vac. Sci. Technol. B 21, 2760 (2003).

${ }^{6}$ U. Plachetka, M. Bender, A. Fuchs, M. Otto, B. Vratzov, B. Vratzov, T. Gilnsner, F. Lindner, and H. Kurz, Microelectron. Eng. 73-74, 167 (2004).

${ }^{7}$ T. Bailey, B. Smith, B. J. Choi, M. Colburn, M. Meissl, S. V. Sreenivasan, J. G. Ekerdt, and C. G. Willsona, J. Vac. Sci. Technol. B 19, 2806 (2001).

${ }^{8}$ T. Bailey, B. J. Choi, M. Meissl, S. Shaya, J. G. Ekerdt, S. V. Sreenivasan, and C. G. Willson, J. Vac. Sci. Technol. B 18, 3572 (2000).

${ }^{9}$ C. Gourgon, C. Perret, J. Tallal, F. Lazzarino, S. Landis, O. Joubert, and R. Pelzer, J. Phys. D 38, 70 (2005).

${ }^{10}$ J. H. Chang and S. Y. Yang, Microsyst. Technol. 10, 76 (2003).

${ }^{11}$ H. Tan, L. Kong, M. Li, C. Steere, and L. Koecher, SPIE's The International Society for Optical Engineering: Emerging Lithographic Technologies VIII, 2004, pp. 213-221.

${ }^{12}$ P. Ruchhoeft, M. Colburn, B. Choi, H. Nounu, S. Johnson, T. Bailey, S. Damle, M. Stewart, J. G. Ekerdt, S. V. Sreenivasan, J. C. Wolfe, and C. G. Willson, J. Vac. Sci. Technol. B 17, 2965 (1999).

${ }^{13}$ K. E. Paul, M. Prentiss, and G. M. Whitesides, Adv. Funct. Mater. 13, 259 (2003).

${ }^{14}$ W. M. Choi and O. O. Park, Nanotechnology 15, 1767 (2004). 